\title{
Clinical and laboratory features of intestinal tuberculosis
}

This article was published in the following Dove Press journal: Clinical and Experimental Gastroenterology

\section{Bhumit Patel' \\ Vipul D Yagnik²}

'Department of Medical Gastroenterology, Akshar Bhoomi Liver and Gastro Care, Ahmedabad, Gujarat, India; ${ }^{2}$ Department of Surgical Gastroenterology, Ronak Endolaparoscopy and General Surgical Hospital, Patan, Gujarat, India

\section{Video abstract}

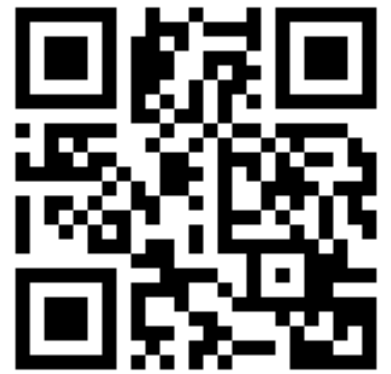

Point your SmartPhone at the code above. If you have a $Q R$ code reader the video abstract will appear. Or use: http://youtu.be/FbsRQcmi40I
Correspondence: Vipul D Yagnik 77 Siddhraj Nagar, Rajmahal Road, Patan 384265, Gujarat, India Email vipul.yagnik@gmail.com
Background/aims: As increasing numbers of Crohn's disease (CD) cases are being recognized in India, so the differential diagnosis of CD and gastrointestinal tuberculosis (GITB) is becoming increasingly important. If patients are misdiagnosed with GITB, toxicity may result from unnecessary anti-TB therapy and treatment of the primary disease (ie, CD) gets delayed. We therefore aimed to assess the accuracy of various parameters that can be used to predict GITB diagnosis at index evaluation.

Materials and methods: This was a prospective, unicentric, observational study carried out in the gastroenterology department of a tertiary care hospital between August 2011 and January 2013. Patients who presented to our hospital and were suspected of having GITB were included in our study. Patients were then followed up over a 6-month period.

Statistical analysis: Chi-square test was used to analyze the data.

Results: Of the 69 patients with GITB, 49 (71.01\%) had thickening of the involved part of the colon and 33 (47.83\%) had abdominal lymphadenopathy. The ileocecal valve was involved in 58 patients $(84.05 \%)$ Histological detection of granulomas had $78.95 \%$ specificity, $36.23 \%$ sensitivity, and $51.40 \%$ accuracy. Tuberculosis polymerase chain reaction was found to have $78.95 \%$ specificity, $71.01 \%$ sensitivity, and $73.83 \%$ accuracy. BACTEC-MGIT culture was found to have $100 \%$ specificity, $20.29 \%$ sensitivity, and $48.60 \%$ accuracy.

Conclusion: Although histology is helpful in ruling out other conditions, TB-specific findings such as caseating granuloma and acid-fast bacilli are rarely seen. Instead, tuberculosis polymerase chain reaction has the highest diagnostic accuracy followed by BACTEC culture.

Keywords: gastrointestinal tuberculosis, colonoscopy, TB polymerase chain reaction, BACTEC culture, antituberculous treatment, biopsy

\section{Introduction}

The incidence of abdominal tuberculosis (TB) has been steadily increasing worldwide over the past 20 years, ${ }^{1-4}$ including in India where gastrointestinal tuberculosis (GITB) is very common. However, incidences of Crohn's disease (CD) are also being increasingly reported throughout the country. ${ }^{5-8}$ Both GITB and CD are granulomatous diseases of the intestine and have a close resemblance in terms clinical, radiological, endoscopic, surgical, and histological features. Thus, differential diagnosis of these 2 conditions remains a major challenge to clinicians. , $^{3,9-16}$

As increasing numbers of CD cases are being recognized in India, differential diagnosis of CD and GITB is becoming increasingly important. ${ }^{10,12}$ If patients are misdiagnosed with GITB, toxicity may result from unnecessary anti-TB therapy (ATT) and treatment of the primary disease (ie, $C D)$ gets delayed. Conversely, the administration 
of steroids alone for CD treatment in the event where GITB is overlooked can be highly detrimental to patients. Currently, patient response to empirical ATT administration is considered an important factor in whether a final diagnosis of TB is made. This poses a further problem as it encourages the emergence of multidrug-resistant TB strains, decreasing drug efficacy in patients who do have TB. These situations highlight the need to definitively diagnose either GITB or CD before any form of empirical treatment is started.

We therefore aimed in our study to assess the accuracy of various parameters that can be used to predict GITB diagnosis at index evaluation.

\section{Materials and methods Study design}

This was a prospective, unicentric, observational study carried out in the gastroenterology department of a tertiary care hospital between August 2011 and January 2013. The study was commenced after receiving approval and clearance from the hospital ethics committee of the Lokmanya Tilak Municipal General Hospital and Medical College, Sion, Mumbai. Written informed consent was obtained from all patients.

\section{Patient evaluation}

Patients who presented to our hospital and were suspected of having GITB were included in our study. Totally, 69 patients were included in the study. The following parameters were prospectively evaluated: patient characteristics and clinical symptoms, gastrointestinal morphology as assessed by colonoscopy, radiological findings on contrast-enhanced computed tomography (CECT) scans of the abdomen, histological findings, and microbiology of involved intestinal segments. Patients were then followed up over a 6-month period, during which they were evaluated at regular intervals to assess the clinical and laboratory parameters. Patients were diagnosed with GITB if they responded favorably to TB treatment.

\section{Patient characteristics and clinical features}

Patient characteristics that were recorded included age and sex. Clinical features that were noted included symptom duration, abdominal pain, weight loss, fever, chronic diarrhea, features of intestinal obstruction, ascites, bloody stools, and extraintestinal disease manifestations.

\section{Colonoscopic evaluation}

The morphology of the colon and ileum were evaluated during colonoscopy. All subjects underwent a colonoscopy and were evaluated for the presence of lesions in the colon and terminal ileum. Lesions were classified as either lesions with ileocecal involvement, patulous ileocecal valves (Figures 1-3), transverse ulcers (Figure 4), nodular lesions, strictures, or skip lesions. The site of involvement was also identified. Images of the healthy intestines are provided for comparison (Figure 5: normal terminal ileum and Figure 6: normal ileocecal valve with cecum).

When a lesion was identified, a minimum of 6 biopsy samples were obtained from the edges and base of the lesion. If lesions were not identified, but abdominal CECT scans suggested thickening of a segment, biopsy samples from the involved segment were taken. If abdominal lymphadenopathy was found, CECTguided biopsy of the lymph nodes was performed.

\section{Radiological evaluation}

Thickening of involved segments of intestine, the presence of strictures, abdominal lymphadenopathy, and other features were noted during evaluation of abdominal CECT scans.

\section{Histological evaluation}

Paraffin-embedded tissue specimens were stained with hematoxylin and eosin. Histological findings were then

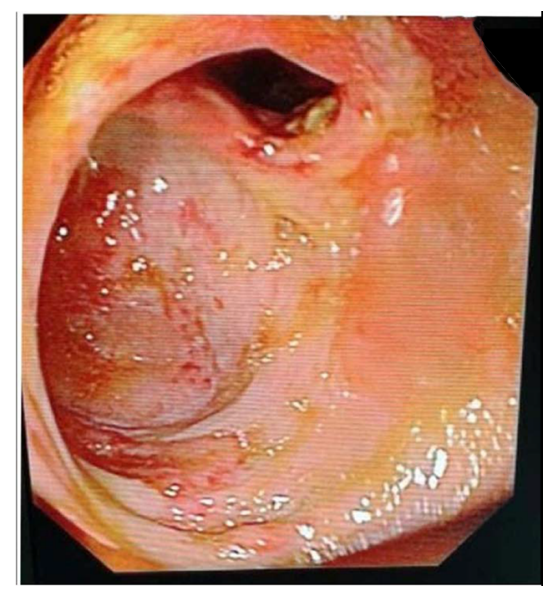

Figure I Patulous ileocecal valve.

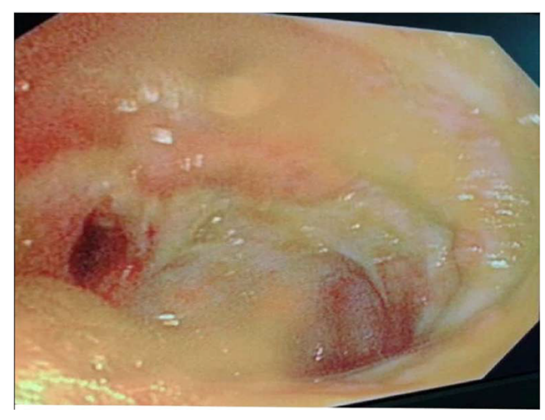

Figure 2 Patulous ileocecal valve with transverse ulcer. 


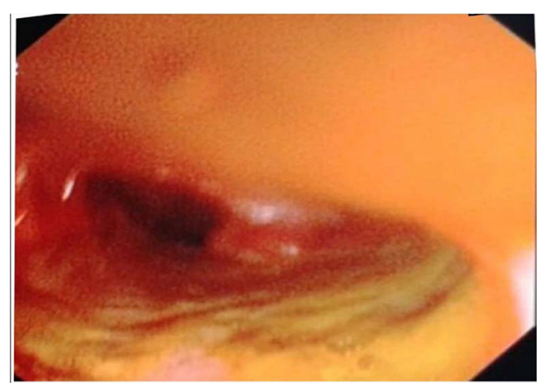

Figure 3 Patulous ileocecal valve with inflamed terminal ileum.

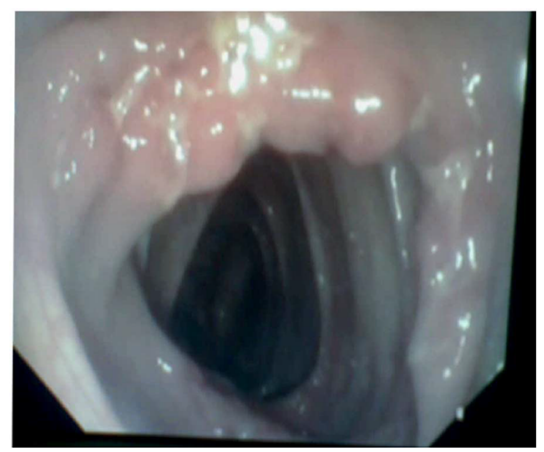

Figure 4 Transverse ulcer at ileocecal valve.

reported by an experienced gastrointestinal pathologist, who was completely unaware of clinical, morphological, and microbiological findings. The following histological features were noted: the presence of granuloma, the characteristics of granuloma (caseation or confluence), ulceration of the surface epithelium, the presence of lymphoid aggregates, and the site and type of inflammatory infiltrate.

\section{Microbiological findings}

Biopsy specimens were smeared for acid-fast bacilli (AFB) staining using the Ziehl-Neelsen technique. Biopsy culture with the BACTEC-MGIT system (BD, Franklin Lakes, NJ, USA) and TB polymerase chain reaction (TBPCR) were also performed for the detection of Mycobacterium tuberculosis.

\section{Diagnostic criteria}

In the presence of a demonstrable lesion in the gastrointestinal tract, a definitive GITB diagnosis was made if specimens fulfilled one or more of the following criteria: ${ }^{17} 1$ ) presence of granuloma upon histological examination of diseased tissue, 2) AFB-positive smears or histological sections, 3) AFB-positive BACTEC-MGIT cultures, and/or 4) AFBpositive TB PCR results. Complete resolution of symptoms after completion of standard ATT was also regarded as a definitive criterion for GITB.

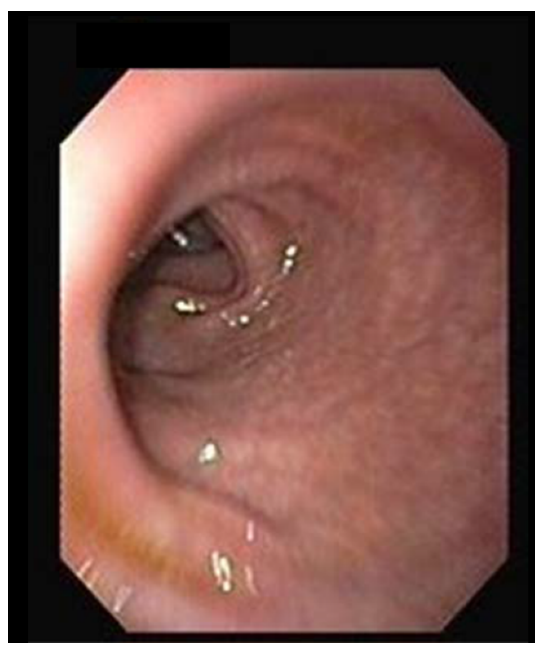

Figure 5 Normal terminal ileum.

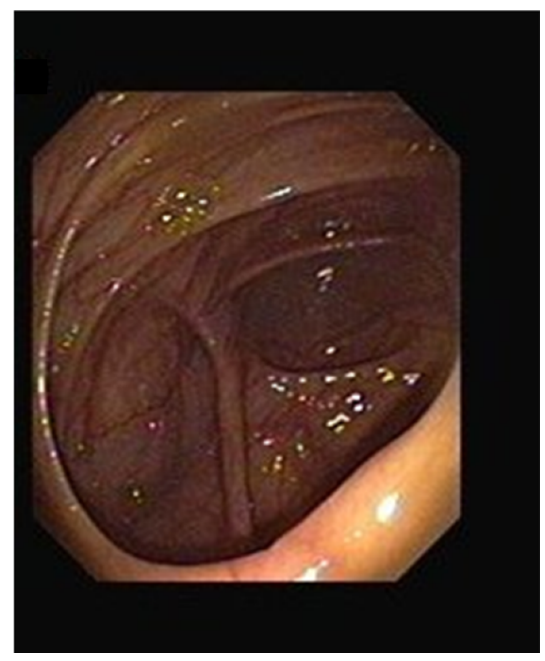

Figure 6 Normal ileocecal valve with cecum.

\section{Statistical analysis}

Data are expressed as mean \pm SD or as numbers and percentages. Chi-square test was used to analyze the data. A $P$-value $<0.05$ was considered statistically significant.

\section{Results}

\section{Patient demographics}

Sixty-nine patients with a mean age of $33.72 \pm 7.88$ years presented with GITB. Of these patients, 43 were male and 26 were female (male to female ratio, 1.65:1). The median duration of GITB was $8.4 \pm 3.7$ months.

\section{Clinical features of patients with GITB}

The clinical characteristics of patients with GITB and the strength of association of these characteristics with GITB 
are shown in Table 1. The clinical features most strongly associated with a diagnosis of GITB were the presence of ascites and presentation with subacute intestinal obstruction.

\section{Endoscopic features of patients with GITB}

Sites within the gastrointestinal tract that were commonly found to be involved in our 69 GITB patients were (in descending order of prevalence) the ileocecal valve in 58 patients $(84.05 \%)$, the ileum in 25 patients $(36.23 \%)$, the ascending colon in 22 patients $(31.88 \%)$, the transverse colon in 4 patients $(5.80 \%)$, and the descending colon in 2 patients $(2.90 \%)$. No patients were found to have GITB with involvement of the sigmoid colon, rectum, or the anal and perianal region.

Lesions detected during endoscopy were also characterized and noted, with their features summarized in Table 2. Of these lesions, transverse ulcers and patulous ileocecal valves were found to be significantly associated with a diagnosis of GITB.

\section{Radiological features of patients with GITB}

Of the 69 patients with GITB, 49 (71.01\%) had thickening of the involved part of the colon and 33 (47.83\%) had abdominal lymphadenopathy. Thickening of an involved portion of the colon was found to have $47.37 \%$ specificity, $86.96 \%$ sensitivity, a positive predictive value of $74.77 \%$, a

Table I Clinical features

\begin{tabular}{lll}
\hline Characteristics & GITB, $\mathbf{n = 6 9}(\%)$ & P-value \\
\hline Abdominal pain & $53(76)$ & $>0.05$ \\
Weight loss & $42(60.87)$ & $>0.05$ \\
Fever & $50(72.46)$ & $>0.05$ \\
Chronic diarrhea & $20(28.99)$ & $>0.05$ \\
Constipation & $5(7.25)$ & $>0.05$ \\
Abdominal mass & $7(10.15)$ & $<0.05$ \\
Blood in stool & $10(14.50)$ & $>0.05$ \\
Ascites & $7(10.15)$ & $<0.05$ \\
Subacute intestinal obstruction & $7(10.15)$ & $<0.05$ \\
Extraintestinal manifestation & $10(14.50)$ & $>0.05$ \\
\hline
\end{tabular}

Abbreviation: GITB, gastrointestinal tuberculosis.

Table 2 Types of lesions

\begin{tabular}{lll}
\hline Endoscopic findings & $\mathbf{n = 6 9}(\%)$ & $\boldsymbol{P}$-value \\
\hline Transverse ulcer & $40(57.97)$ & $<0.05$ \\
Patulous ileocecal valve & $20(28.99)$ & $<0.05$ \\
Nodularity & $15(21.74)$ & $>0.05$ \\
Stricture & $6(8.70)$ & $>0.05$ \\
Skip lesions & $5(7.25)$ & $>0.05$ \\
\hline
\end{tabular}

negative predictive value of $66.67 \%$, and $72.90 \%$ accuracy. For abdominal lymphadenopathy, these values were $36.84 \%$, $66 \%, 47.83 \%, 55.26 \%$, and $50.46 \%$, respectively.

\section{Histological features of patients with GITB}

Of the 69 patients diagnosed with GITB, granulomas were found in the stained biopsy sections of 49 patients $(71.01 \%)$. Histological detection of granulomas had $78.95 \%$ specificity, $36.23 \%$ sensitivity, a positive predictive value of $75.76 \%$, a negative predictive value of $40.54 \%$, and $51.40 \%$ accuracy.

\section{TBPCR results in patients with GITB}

Of the 69 patients with GITB, TB PCR was positive for M. tuberculosis in 49 (71.01\%). TBPCR was found to have $78.95 \%$ specificity, $71.01 \%$ sensitivity, a positive predictive value of $85.96 \%$, a negative predictive value of $60 \%$, and $73.83 \%$ accuracy.

\section{BACTEC-MGIT culture results in patients with GITB}

BACTEC-MGIT cultures established from colonoscopic biopsy specimens were positive for M. tuberculosis in 14 of our $69(20.29 \%)$ GITB patients. BACTEC-MGIT culture was found to have $100 \%$ specificity, $20.29 \%$ sensitivity, a positive predictive value of $100 \%$, a negative predictive value of $40.86 \%$, and $48.60 \%$ accuracy.

A comparison of all the diagnostic modalities used and their sensitivities, specificity, etc. is shown in Table 3.

\section{Discussion}

TB can affect any part of the gastrointestinal tract from the mouth to the anus, as well as the pancreatobiliary system. Its symptoms can also mimic those of various other intestinal disorders, both common and rare. As intestinal infections occur at a high rate in the Asia-Pacific region, the area was previously thought to have a low incidence rate of inflammatory bowel disorder (IBD), though this could also be due to a lack of population-based studies and low diagnostic awareness of IBD. However, current literature and recent information suggest that a true increase of IBD is occurring throughout this region. ${ }^{5}$ At the same time, the incidence of TB is also rising elsewhere, including in both the US and the UK. ${ }^{3,18}$

Two disorders that present similarly in terms of both clinical features and colonoscopy findings are CD and tuberculous colitis. Despite their similar features, however, their pathogenesis and therefore treatment differ. Appropriate 
Table 3 A comparison of all the diagnostic modalities used and their sensitivities, specificity

\begin{tabular}{llllll}
\hline Parameters & Specificity (\%) & Sensitivity (\%) & PPV (\%) & NPV (\%) & Accuracy (\%) \\
\hline Colonoscopic finding & 65.79 & 100 & 84.15 & 100 & 87.85 \\
CECT Abdomen & 47.37 & 86.96 & 74.77 & 66.67 & 72.90 \\
Abdominal lymphadenopathy & 36.84 & 66 & 47.83 & 55.26 & 50.46 \\
BACTEC & 100 & 20.29 & 100 & 40.86 & 48.60 \\
Granuloma on histology & 78.95 & 36.23 & 75.76 & 40.54 & 51.40 \\
TBPCR positive & 78.95 & 71.01 & 85.96 & 60 & 73.83 \\
Complete response to ATT & 100 & 100 & 100 & 100 & 100 \\
\hline
\end{tabular}

Abbreviations: ATT, anti-TB therapy; CECT, contrast-enhanced computed tomography; NPV, negative predictive value; PPV, positive predictive value; TBPCR, tuberculosis polymerase chain reaction.

ATT administration leads to complete cure in most cases of tuberculous ileocolitis, whereas $\mathrm{CD}$ is a progressive relapsing illness unaffected by ATT. Differentiating GITB from other disorders is therefore important in its management. In this study, we studied patients who were suspected to have GITB and assessed the accuracy of various diagnostic modalities in its diagnosis.

As in previously reported case series,${ }^{19-23}$ the majority of our patients had nonspecific symptoms, with weight loss and abdominal pain being the predominant symptoms in over $70 \%$ of patients. Around half of the patients in this study also presented with a fever. In contrast, the detection of abdominal masses by physical examination was noted in only $10.15 \%$ of our patients. This proportion is significantly lower than that in the study by Palmer et al, ${ }^{24}$ in which $43 \%$ patients developed an abdominal mass comprising either inflammatory intestinal lesions or enlarged mesenteric lymph nodes. This difference is likely to be because small bowel involvement was predominant in Palmer et al's study ${ }^{24}$ (occurring in 40 of 42 patients), with only $4.7 \%$ of patients showing isolated TB affecting the colon. Rectal bleeding was also observed in $14.55 \%$ of our patients. In Mukewar et al's study, ${ }^{23}$ it was observed in $20 \%$ of patients, and in Mishra et al's study, ${ }^{21} 10 \%$ of patients required surgery for massive lower gastrointestinal bleeding. Furthermore, subacute intestinal obstruction was noted in $7(10.15 \%)$ of our patients. This contrasts with Mukewar et al's study, ${ }^{23}$ in which $44.8 \%$ of patients had luminal narrowing as detected by index colonoscopy. We also found that narrowing resolved with anti-TB treatment, suggesting that these strictures were inflammatory. Only 1 patient required surgical intervention.

The present study also confirms published data demonstrating that the ileocecal region is most often affected in GITB ${ }^{5,25}$ This is probably because it is an area of physiological stasis, is a site of avid water and electrolyte absorption, and has relatively little digestive capacity. These factors permit prolonged contact between bacilli and mucosa. ${ }^{1,18}$
In addition, the predominant lesion types identified during colonoscopy in our study were transverse ulcerations and patulous ileocecal valves, which is in line with some previously published studies. ${ }^{19-23}$ These lesions were predominantly located on the right side of the colon (ie, the ileocecal valve, cecum, and ascending colon). Nagi et al, ${ }^{26}$ on the other hand, found that the transverse colon was the most commonly affected site and that strictures were the predominant lesion type. However, these findings were radiological rather than colonoscopic. Indeed, the first systematic prospective analysis of endoscopic findings in GITB and CD patients found that the presence of 4 endoscopic GITB features (namely, transverse ulcers, pseudopolyps, the involvement of fewer than four segments, and a patulous ileocecal valve) had a high positive predictive value of $88.9 \%$ for GITB. ${ }^{27} \mathrm{~A}$ study involving Chinese patients developed a mathematical regression equation including the following colonoscopic features, involvements of rectum, longitudinal ulcers, transverse ulcers, rodent like ulcers, cobblestone sign, and fixed open ileocecal valve, revealed a sensitivity and specificity of $82.9 \%$ and $82.0 \%$, respectively. ${ }^{28}$ In a South African study that compared CD with GITB patients, the histolopathological features that were only detected in cases with GITB had confluent granulomata, 10 or more granulomata per biopsy site and caseous necrosis. ${ }^{29}$

With regard to radiological features, we found that bowel wall thickening and lymphadenopathy were found in $79.71 \%$ and $47.82 \%$ of patients, respectively. On the other hand, a study by Sinan et $a l^{30}$ found bowel wall thickening in $38 \%$ of patients and lymphadenopathy in $46.9 \%$. This discrepancy is likely to be because Sinan et al's study ${ }^{30}$ was based on CT scan findings from abdominal TB patients with high rates of peritoneal involvement. Despite this difference, both our and Sinan et al's ${ }^{30}$ results suggest that radiological features do not reliably predict GITB diagnosis.

Other routine tests for TB, such as AFB smear tests with the conventional Ziehl-Neelsen stain, traditional AFB culture 
using egg-based or agar-based media, and guinea pig inoculation lack sensitivity and are time-consuming. ${ }^{31}$ In contrast, smear tests using fluorescence staining and culture with the BACTEC technique are more rapid and sensitive. ${ }^{31}$ As GITB is a paucibacillary disease, however, the sensitivity of these methods for detecting $M$. tuberculosis in clinical specimens remains poor. Furthermore, although many serological tests for TB are commercially available, they are not satisfactory when a differential diagnosis needs to be made. ${ }^{31}$

Recently, Pulimood et $\mathrm{al}^{32}$ reported that in addition to AFB detection, biopsy features suggestive of TB include confluent granulomas, a lymphoid cuff around granulomas, granulomas larger than $400 \mu \mathrm{m}$ in diameter, 5 or more granulomas in biopsies from 1 segment, granulomas located in the submucosa or in granulation tissue (often as palisaded epithelioid histiocytes), and disproportionate submucosal inflammation. In our study, we collected mucosal biopsies during colonoscopy. However, the usefulness of mucosal biopsies is limited because granulomas, the primary feature that differentiates TB from CD, are found in only $50 \%-80 \%$ of intestinal mucosal biopsies from patients with clinically confirmed TB. ${ }^{33-35}$ Furthermore, caseation of granulomas and AFB, the diagnostic features of TB, are, respectively, found in only $18 \%-33 \%{ }^{19,35}$ and as low as $5 \%$ of cases. ${ }^{19}$ Indeed, we found that mucosal biopsies showing caseation of granulomas could predict TB diagnosis with $51.40 \%$ accuracy, which is not sufficient for differentially diagnosing GITB and CD.

$\mathrm{Du}$ et $\mathrm{al}^{36}$ published a meta-analysis including 10 randomized trials in PLoS One showing that the sensitivity and specificity of confluent granulomas for differentiation of GITB from CD were 38\% and 99\%, respectively, while those of epithelioid histiocytes in ulcer bases were $41 \%$ and $94 \%$, respectively. ${ }^{36}$

For culture-based TB diagnosis, the BACTEC 460TB system works on the principle of early specific detection of mycobacterial growth. ${ }^{37}$ This metabolism-based diagnostic system is advantageous as its speed of diagnosis is double that of conventional Lowenstein-Jensen medium; while a culture observation period of 6 weeks is required when LowensteinJensen medium is used, it is possible to provide a negative report in 3 weeks when the BACTEC 460TB system is used. In our study, a positive BACTEC culture was observed in $20.29 \%$ of TB patients and had $48.60 \%$ accuracy. The rate of culture positivity was higher in Shah et al's study, ${ }^{38}$ where $76 \%$ of TB patients showed positive BACTEC 460TB cultures. This difference may be because while all cultures were established in our study using colonoscopic biopsies, Shah et al's ${ }^{38}$ samples were drawn from a range of procedures including colonoscopic, laparoscopic, and open surgical biopsies.
Finally, amplification of mycobacterial DNA sequences by PCR, which is a rapid and accurate diagnostic method, has been shown to be more promising for mycobacterial detection in clinical specimens. Indeed, we found that TBPCR had an accuracy of $73.83 \%$ in the diagnosis of GITB. This is within the range of previous studies, including a study by Amarapurkar et al, ${ }^{39}$ where TBPCR had a diagnostic accuracy of over $80 \%$, and a study by Gan et al, ${ }^{40}$ where $64.1 \%$ of TB patients had a positive TBPCR result.

\section{Limitations of the study}

1) This study is not a comparative study between GITB and $\mathrm{CD}$ which, given the minimal incidence of $\mathrm{CD}$, would be difficult. 2) We mentioned $C D$ as the background motivation for study, not as a component of research question.

\section{Conclusion}

Patients with GITB tend to present with nonspecific symptoms. Hence, diagnosis in countries where TB is nonendemic involves a high index of suspicion (migrant population, immunosuppressed individuals). While abdominal pain and weight loss are the predominant symptoms, ulceration, patulous ileocecal valves, and luminal narrowing are the prominent endoscopic findings and appear to mostly affect the right colon. Although histology is helpful in ruling out other conditions, TB-specific findings such as caseating granuloma and AFB are rarely seen. Instead, TBPCR has the highest diagnostic accuracy followed by BACTEC culture. Furthermore, the majority of colonic lesions, including strictures, resolve with anti-TB treatment, suggesting that strictures are inflammatory rather than fibrotic. Follow-up colonoscopy is not required in those who have symptomatic improvement after anti-TB treatment.

\section{Acknowledgment}

We would like to acknowledge the help provided by Dr Sushil Dawka (Professor of Surgery, Mauritius) for his kind help in revising the manuscript.

\section{Author contributions}

This work was carried out in collaboration between both authors. Author Bhumit Patel designed the study, performed the statistical analysis, wrote the protocol, and wrote the first draft of the manuscript. Author Vipul Yagnik managed the analyses of the study, and critically revised the manuscript. Both authors contributed toward data analysis, drafting and critically revising the paper and agree to be accountable for all aspects of the work. 


\section{Disclosure}

The authors report no conflicts of interest in this work.

\section{References}

1. Guth AA, Kim U. The reappearance of abdominal tuberculosis. Surg Gynecol Obstet. 1991;172(6):432-436.

2. Watson JM, Gill ON. HIV infection and tuberculosis. BMJ. 1990;300(6717):63-65.

3. Marshall JB. Tuberculosis of the gastrointestinal tract and peritoneum. Am J Gastroenterol. 1993;88(7):989-999.

4. Snider DJ, Roper WL. The new tuberculosis. N Engl J Med. 1992; 326(10):703-705.

5. Ouyang Q, Tandon R, Goh KL, Ooi CJ, Ogata H, Fiocchi C. The emergence of inflammatory bowel disease in the Asian Pacific region. Curr Opin Gastroenterol. 2005;21(4):408-413.

6. Jayanthi V, Robinson RJ, Malathi S, et al. Does Chron's disease need differentiation from tuberculosis? J Gastroenterol Hepatol. 1996;11(2):183-186.

7. Bhansali SK. Abdominal tuberculosis. Experiences with 300 cases. Am J Gastroenterol. 1977;67(4):324-337.

8. Chung KM, Kim HS, Park SY, et al. The changes in incidence of Chron's disease and intestinal tuberculosis in Korea. Korean J Gastroenterol. 2008;52(6):351-358.

9. Amarapurkar D, Patel N. Crohn's disease in India. Gastroenterol Today. 2002;6:73-75.

10. Pulimood AB, Ramakrishna BS, Kurian G, et al. Endoscopic mucosal biopsies are useful in distinguishing granulomatous colitis due to Crohn's disease from tuberculosis. Gut. 1999;45(4):537-541.

11. Pai CG, Khandige GK. Is Crohn's disease rare in India? Indian J Gastroentero. 2000;19(1):17-20.

12. Patel N, Amarapurkar DN, Agal S, et al. Gastrointestinal luminal tuberculosis: establishing the diagnosis. Gastroenterol Hepatol. 2004;19(11):1240-1246.

13. Ouyang Q. Inflammatory bowel disease in China. J Gastroenterol Hepatol. 2000;15:S25.

14. Das P, Shukla HS. Clinical diagnosis of abdominal tuberculosis. $\mathrm{Br} J$ Surg. 1976;63:941-946.

15. Anand BS. Distinguishing Crohn's disease from intestinal tuberculosis. Nat Med J Ind. 1989;2:172-175.

16. Chuttani HK, Sarin SK. Intestinal tuberculosis. Ind J Tuber. 1985;32: $117-125$

17. Amarapurkar DN, Patel ND, Rane PS. Diagnosis of Crohn's disease in India where tuberculosis is widely prevalent. World $J$ Gastroenterol. 2008;14(5):741-746.

18. Hayward AC, Watson JM. Tuberculosis in England and Wales 19821993: notifications exceeded predictions. Commun Dis Rep CDR Rev. 1995;5(3):R29-R33.

19. Alvares JF, Devarbhavi H, Makhija P, Rao S, Kottoor R. Clinical, colonoscopic, and histological profile of colonic tuberculosis in a tertiary hospital. Endoscopy. 2005;37(4):351-356.

20. Villanueva SE, Martinez HMP, Fernando ATFJ, Valdés OM. Colonic tuberculosis. Dig Dis Sci. 2002;47(9):2045-2048.

21. Misra SP, Misra V, Dwivedi M, Arora JS, Kunwar BK. Tuberculous colonic strictures: impact of dilation on diagnosis. Endoscopy. 2004;36(12):1099-1103.

Clinical and Experimental Gastroenterology

\section{Publish your work in this journal}

Clinical and Experimental Gastroenterology is an international, peerreviewed, open access, online journal publishing original research, reports, editorials, reviews and commentaries on all aspects of gastroenterology in the clinic and laboratory. This journal is included on PubMed. The manuscript management system is completely online
22. Chong VH, Lim KS. Gastrointestinal tuberculosis. Singapore Med J. 2009;50(6):638-645; quiz 646.

23. Mukewar S, Mukewar S, Ravi R, Prasad A, Dua KS. Colon tuberculosis:endoscopic feature and prospective endoscopic follow up after anti tuberculous treatment. Clin Transl Gastroenterol. 2012; 3(10):e24.

24. Palmer KR, Patil DH, Basran GS, Riordan JF, Silk DB. Abdominal tuberculosis in urban Britain - a common disease. Gut. 1985;26: 1296-1305.

25. Misra SP, Misra V, Dwivedi M, Gupta SC. Colonic tuberculosis: clinical features, endoscopic appearance and management. $J$ Gastroenterol Hepatol. 1999;14(7):723-729.

26. Nagi B, Kochhar R, Bhasin DK, Singh K. Colorectal tuberculosis. Eur Radiol. 2003;13(8):1907-1912.

27. Lee YJ, Yang SK, Byeon JS, et al. Analysis of colonoscopic findings in the differential diagnosis between intestinal tuberculosis and Crohn's disease. Endoscopy. 2006;38(6):592-597.

28. Li X, Liu X, Zou Y, et al. Predictors of clinical and endoscopic findings in differentiating Crohn's disease from intestinal tuberculosis. Dig Dis Sci. 2011;56:188-196.

29. Kirsch R, Pentecost M, Hall PM, et al. Role of colonoscopic biopsy in distinguishing between Crohn's disease and intestinal tuberculosis. J Clin Pathol. 2006;59:840-844.

30. Sinan T, Sheikh M, Ramadan S, Sahwney S, Behbehani A. Ct features in abdominal tuberculosis: 20 years experience. BMC Med Imaging. 2002;2(1):3.

31. Woods GL. The mycobacteriology laboratory and new diagnostic techniques. Infect Dis Clin North Am. 2002;16(1):127-144.

32. Pulimood AB, Amarapurkar DN, Ghoshal U, et al. Differentiation of Crohn's disease from intestinal tuberculosis in India in 2010. World $J$ Gastroenterol. 2011;17(4):433-443.

33. Makharia GK, Srivastava S, Das P, et al. Clinical, endoscopic, and histological differentiations between Crohn's disease and intestinal tuberculosis. Am J Gastroenterol. 2010;105(3):642-651.

34. Pettengell KE, Larsen C, Garb M, Mayet FG, Simjee AE, Pirie D Gastrointestinal tuberculosis in patients with pulmonary tuberculosis. Q J Med. 1990;74(275):303-308.

35. Shah S, Thomas V, Mathan M, et al. Colonoscopic study of 50 patients with colonic tuberculosis. Gut. 1992;33(3):347-351.

36. Du J, Ma YY, Xiang H, Li YM. Confluent granulomas andulcers lined by epithelioid histiocytes: new ideal method for differentiation of ITB and CD? A meta analysis. PLoS One. 2014;9:e103303.

37. Katoch VM. Newer diagnostic techniques for tuberculosis. Indian $J$ Med Res. 2004;120:418-428.

38. Shah SR, Shenai S, Desai DC, Joshi A, Abraham P, Rodrigues C. Comparison of Mycobacterium tuberculosis culture using liquid culture medium and Lowenstein Jensen medium in abdominal tuberculosis. Indian J Gastroenterol. 2010;29(6):237-239.

39. Amarapurkar DN, Patel ND, Amarapurkar AD, Agal S, Baigal R, Gupte P. Tissue polymerase chain reaction in diagnosis of intestinal tuberculosis and Crohn's disease in India. J Assoc Physicians India. 2004;52:863-867.

40. Gan HT, Chen YQ, Ouyang Q, Bu H, Yang XY. Differentiation between intestinal tuberculosis and Crohn's disease in endoscopic biopsy specimens by polymerase chain reaction. Am J Gastroenterol. 2002;97(6):1446-1451. 